INVESTIGACIONES

\title{
ESTUDIO DE CASO DE LA COHERENCIA INTERNA DE PROGRAMAS DE ASIGNATURAS EN SEIS CARRERAS QUE PROMUEVEN COMPETENCIAS DE LA UNIVERSIDAD AUSTRAL DE CHILE
}

\author{
A Case Study about the Internal Coherence of the Programs \\ of Subject Matters of six Majors that promote Competencies \\ at the Universidad Austral de Chile
}

\begin{abstract}
Um estudo de caso sobre a Coerência de Programas de matérias em seis carreiras que promovem competências da Universidade Austral do Chile
\end{abstract}

\author{
Myriam Márquez Manzano*1, Jacquelinne Sandoval Machuca**2; \\ María Cristina Torres Andrade***3, Sergio Pavié Coronado****4 \\ *Universidad Austral de Chile myriam.marquez@docentes.uach.cl (056-063-221423) \\ **Académica de la Dirección de Postgrado de la Universidad Metropolitana \\ de Ciencias de la Educación. jxsandov@gmail.com (056-09-7707062) \\ ***Académica del Instituto de Salud Pública de la Universidad Austral de Chile \\ mtorres1@uach.cl (056-063-63 221141) \\ ****Académico del Instituto de Salud Sexual y Reproductiva, \\ de la Universidad Austral de Chile (056-063-221423)
}

\section{RESUMEN}

Se discuten los resultados de la primera fase de la investigación DID S-2007-075 que se desarrolla en la UACh. Se indaga la coherencia interna de los programas de asignaturas en seis carreras acreditadas. Coherencia interna es la articulación lógica entre los objetivos o competencias a lograr, las metodologías para su desarrollo y la evaluación para certificar logro. El Índice de Coherencia Interna pesquisa que de los 359 programas revisados sólo el 20\% de ellos alcanza el puntaje para ser considerado coherente con el modelo curricular orientado al logro competencias. Competencias explícitas promovidas son la Capacidad de aplicar los conocimientos en la práctica (46\%); Conocimiento sobre el área de estudio y la profesión (44\%) y la Capacidad de abstracción, análisis y síntesis $(43 \%)$.

Palabras clave: Programas de asignaturas, currículo, competencias genéricas.

1 Matrona Universidad Austral de Chile-Magíster en Educación con mención en Evaluación Universidad de La Frontera-Candidata a Doctora en Ciencias de la Educación, Pontificia Universidad Católica de Chile. Profesora Auxiliar del Instituto de Salud Sexual y Reproductiva.

2 Educadora-Magíster en Educación con mención en Currículo Universidad Metropolitana de Ciencias de la Educación-Candidata a Doctora en Ciencias de la Educación, Pontificia Universidad Católica de Chile.

3 Enfermera y Matrona Universidad Austral de Chile-Magíster en Desarrollo Rural, Universidad Austral de Chile. 4 Matrón, Universidad Austral de Chile-Epidemiólogo Clínico, Universidad de La Frontera.

Notas de los autores: En consideración al lenguaje de género, los autores reconocen explícitamente diferencias entre lo femenino y lo masculino, sin embargo, en atención a la optimización de la lectura del artículo, se utilizará el género masculino como el incluyente de ambos. 


\begin{abstract}
The results of the first stage of the research DID S-2007-07 conducted at the UACh are discussed. An inquiry was made about the degree of internal coherence of programs of subject matters of six accredited majors. Internal coherence is the logical coordination between the objectives or competencies to be achieved, the methodologies used for their development and the assessment system to certify the attainment. The Internal Coherence Ratio showed that only $20 \%$ of the 359 programs of subject matters revised ranked as coherent with the curricular model oriented towards the achievement of competencies. Explicit competencies fostered were the ability to apply theoretical knowledge in praxis (46\%); the knowledge about the field of study and the profession (44\%), and the capacity of abstraction, analysis and synthesis (43\%).
\end{abstract}

Key words: Subject matter programs, curriculum, generic competencies.

\title{
RESUMO
}

Discutimos os resultados da primeira fase da investigação DID S-2007-07 [1], feita na UACh. Explora-se a coerência interna dos programas de curso em seis carreiras acreditadas. Por coerência interna entende-se a articulação lógica entre os objetivos ou as competências a atingir, as metodologias para a o seu desenvolvimento e a avaliação a fim de certificar sua realização. O Índice de coerência interna verificou que dos 359 programas examinados, apenas $20 \%$ alcançaram a pontuação para ser considerados compatíveis com o currículo modelo, com vista a atingir competências. As competências explicitamente mais promovidas são a capacidade de aplicar os conhecimentos na prática (46\%); os conhecimentos sobre a área de estudo e da profissão (44\%), e a capacidade de abstração, análise e síntese (43\%).

Palabras-chave: Programas de curso, currículo, competências genéricas.

\section{INTRODUCCION}

La aparición en el escenario universitario chileno de los proyectos del Programa de Mejoramiento de la Calidad y la Equidad en la Educación Superior (MECESUP) configuró un movimiento de "ebullición" curricular en el ámbito académico e instaló en el tapete de la discusión de la academia términos como malla curricular; perfil de egreso; acreditación; competencias; aprendizaje centrado en el estudiante y metodologías activas entre muchos otros conceptos educativos que transformaron los discursos de los académicos como constructores curriculares y movilizaron prácticas diferentes.

A partir de la propuesta generada por el Estado (MECESUP), las casas de estudio superior iniciaron procesos de transformación curricular de diversa intensidad para sintonizar con las demandas estatales. La finalidad de estos cambios apuntaba a cumplir los objetivos del programa: formar recursos humanos de alto nivel; promoción del mejoramiento de la calidad y la eficiencia de la educación superior y, por último, articular el desarrollo de esos objetivos con los procesos de internacionalización teniendo como referentes principales los procesos europeos de educación superior, tanto el acuerdo de Bolonia como el proyecto Tuning (MECESUP, s.f.).

De este modo, la Universidad Austral de Chile (UACh), tal como otras casas de estudio, inició cambios curriculares para acreditar sus carreras por el Consejo Nacional de Acreditación de Pregrado (CNAP), hoy transformada en la CNA. A diferencia de la CNAP, ésta es una entidad autónoma cuyo fin es verificar y promover la calidad de las Universidades, Institutos Profesionales y Centros de Formación Técnica autónomos y de las carreras y programas que ellos ofrecen (CNA, 2009). Este proceso de cambio se acompañó de la capacitación de los académicos, lo que en la experiencia de nuestro grupo de trabajo fue diversa en intensidad, perspectivas teóricas y formas de operacionalizar. 
Esta forma de implementación propició que las propuestas curriculares también fueran disímiles y sus cambios alcanzasen diferentes niveles tanto de profundidad como de apropiación.

Es posible estimar que los grados diferentes de apropiación curricular de los académicos dependieron en parte de la forma y diseño que se utilizó para capacitar, y de los tiempos que fueron asignados para diseñar asignaturas, mallas curriculares y perfiles. El escaso ejercicio de diseño, implementación, desarrollo y evaluación del currículo no promueve el aprendizaje activo ni otorga sentido al quehacer curricular (Vigotzky, 2001 y Ausubel, 1976). Por otro lado, Pozo y cols. (1989) sostienen que para que ocurra el cambio conceptual debe existir insatisfacción con concepciones existentes y la presencia de una nueva concepción que parezca inteligible y plausible. Bajo estos supuestos teóricos es posible asumir que la calidad de lo diseñado pueda ser objeto de cuestionamientos toda vez que la forma de instalación del nuevo modelo curricular no tomó en debida cuenta al Ser del docente.

\section{ALGUNAS PRECISIONES TEORICAS Y CONCEPTUALES}

La irrupción del modelo curricular por competencias significó un replanteamiento del modo en que se construye currículo y de la forma en que los constructores curriculares toman decisiones, proceso nunca antes abordado con la intensidad que hoy se aprecia (Hoogveld, A.; Paas, F., \& Jochems, W., 2005; Márquez, 2007). Esto mismo ha permitido que la escasa literatura que existía sobre el campo del diseño curricular en la educación superior se haya revertido positivamente. Hoy es posible encontrar una amplia producción de literatura e investigación relacionada con currículo, lo que ha permitido la evolución de alguno de sus conceptos. Uno de ellos es el concepto Construcción Curricular que se está entendiendo cada vez más como Gestión Curricular. Este es un concepto ya en uso en el mundo de la Escuela que supone una mirada gerencial a la formación profesional. (MINEDUC, sf). No obstante, en la Escuela, es el nivel central -MINEDUC- el que genera el currículo que luego los docentes implementan y contextualizan. En la Universidad la mirada gerencial a la formación supuso el abandono de la tradicional perspectiva de un proceso dicotómico, estable y descontextualizado de Diseño y Desarrollo (Coll, 1987), para asumir teóricamente una construcción que tiende a la participación activa y masiva de los actores; retroalimentada y centrada en la efectividad del proceso y en el logro óptimo de los productos (Zabalza, 2000).

Uno de los actores externos a la Universidad, pero con fuerte peso en las decisiones a tomar como marco referencial para la formación de profesionales, lo constituyen los empleadores. Por ello, ya es un lugar común en la literatura especializada en educación superior señalar que los cambios curriculares realizados buscan la alineación de la formación profesional con las necesidades cambiantes y emergentes del mercado (Barnett, 2002; Brunner, 2005; Hoogveld A. y cols., 2005; Zabalza, 2000; Aristimuño, 2008). Esta alineación ha sido asumida desde un postura crítica de mayor o menor cuantía o, como bien señala Barnett, de manera reactiva o proactiva. Para muchos la relación universidad y mercado es hoy en día indiscutible y, tal como sostiene Brunner (2007), existe dentro de la misma lógica economicista un "Mercado Universitario" en el cual la gestión de procesos formativos adquiere un rol primordial en la subsistencia y competitividad de los centros. 
Hablar de competencias conlleva hoy -aunque cada vez menos- asumir que se trata de un concepto difuso y sobredimensionado, al que se mira con sospecha, devoción o incluso se le considera una moda transitoria (Sandoval y Márquez, 2008). Sin embargo, los procesos curriculares hacia esta propuesta han incorporado de manera explícita su adscripción al modelo. En el caso de la UACh, esta adscripción toma lugar dentro de los objetivos de su Plan Estratégico (Plan estratégico 2008-2011) y además ha sido operacionalizado en documentos orientadores como el Modelo Educacional y Enfoque Curricular de la UACh (2007) y el texto Orientaciones prácticas para docentes, la Docencia Universitaria bajo un Enfoque de Competencias (Jabif, L., 2007). En este último se establece, bajo los planteamientos de Le Boterf (2000), que la competencia es la movilización y combinación de recursos personales y de redes para la actuación eficaz y entiende por recursos los conocimientos generales, cognitivos, operativos, para el relacionamiento social, los valores y actitudes, y la experiencia y cultura. En tanto, entiende por recursos de redes una amplia gama como las bases de datos, bancos de muestras, bases de proyectos, redes de expertos, redes de cooperación científica, redes de clientes, redes informáticas, observatorios, catálogos de información o procedimientos, diccionario de normas, colecciones (Jabif, 2007:27). Finalmente el decreto 271 del año 2005 define Competencia como "un conjunto integrado y articulado de conocimientos, hábitos, actitudes, valores y destrezas evaluables e interrelacionadas que permiten actuar sobre un aspecto de la realidad personal, social, natural o simbólica y suponen la reflexión sobre el propio aprendizaje" (UACh, 2005, pp. 25-26). Dentro de este mismo documento se señala que las Competencias Genéricas hacen referencia a saberes transversales asociados a desempeños comunes, independientemente de las diversas ocupaciones y profesiones (analizar, interpretar, organizar, investigar, enseñar, planear). Toda esta gama de documentos constituyen los marcos orientadores o prescriptivos, es decir, la demarcación que establece los límites y alcances esperados por la institución.

Con fines de este estudio la coherencia será entendida como la conexión lógica entre las partes de un todo que permiten concebirlo como una unidad en la que cada parte o segmento se articula de modo armónico con los otros. Esta conexión lógica -coherenciaserá motivo de análisis en dos planos: a) en un nivel micro, la coherencia interna de los programas de asignatura corresponde a la conexión lógica al interior de los productos diseñados; esto es, lo que el programa de asignatura propone como relación articulada en tres elementos básicos contenidos en los programas de asignatura: Objetivos o Competencias, Estrategias Metodológicas para favorecer el aprendizaje y Estrategias de Evaluación que certifican el logro y b) entre lo que se diseña como propuesta formativa y lo que luego se lleva a cabo en la implementación y desarrollo de tal propuesta. Es decir, lo que ocurre en el espacio del aula.

A pesar de que la explicitación de competencias es una práctica que ya se ha incorporado en los diseños de planes de estudios, no se aprecia la misma claridad ni distinción en las estrategias metodológicas requeridas para fomentar su desarrollo y mucho menos las estrategias evaluativas que certificarán su logro. Ya en los años 70, cuando McClelland, propuso que los test de selección debían de proveer información más confiable acerca de las características de las personas y no sólo de su nivel de inteligencia, se inició el cambio del foco de la evaluación de la inteligencia, hacia la evaluación de la competencia y esto permitió orientar los currículos en quién está aprendiendo y no en quién está enseñando. Todo ello en el entendido de que los problemas de la medición de aprendizajes, 
implementación de ellos y los requerimientos de recursos docentes: metodológicos, de tiempo y de conexión con el mundo externo de la empresa, aún siguen sin resolverse del todo (Schmal y Ruiz Tagle, 2007).

Como señala Cowan (1998), citado en Knight (2006), la enseñanza es un proceso por el cual todo aquel que lo viva o participe estas situaciones de aprendizaje no puede sino salir aprendiendo. Estas "estrategias metodológicas" corresponden al planteamiento conjunto de las directrices a seguir en cada una de las fases del proceso de enseñanza y aprendizaje, de acuerdo a métodos que permitan esos logros y que consideren algunos de los estilos de aprendizajes de los estudiantes. Zabala y Arnau (2007) van al extremo de señalar que la enseñanza de las competencias es la "antítesis de la enseñanza tradicional". Estas y otras afirmaciones demuestran el escaso valor de la clase expositiva tradicional, porque configura a un receptor pasivo de información, "educación bancaria" en el decir de Freire. Por ello el modelo propone estrategias metodológicas que promoverían el aprendizaje significativo (Smith, D.; Hedley, P. y Molloy, M. 2008; Timmerman, B., Strickland, D. y Carstensen, S., 2008). Un claro ejemplo de ello es la incorporación del Aprendizaje Basado en Problemas; el Diseño de portafolios; el Estudio de casos; los Debates; el trabajo de Proyecto; los Trabajos grupales y Prácticas en terreno o Laboratorios -entre otras- que permiten al estudiante ser el gestor de su propio aprendizaje.

En cuanto a la evaluación debería caracterizarse, en la práctica, como la coherencia entre los postulados y las acciones (Herrera, 2009). Entre lo que se dice y lo que se hace, entre lo deseable y lo posible, y debe estar asociada a un contexto o una evaluación integrada al proceso de enseñanza y aprendizaje. La evaluación de competencias supone a priori que están explicitados los dominios, competencias y subcompetencias en el perfil de egreso, malla curricular y programas de asignaturas. De este modo se logra la coherencia entre los modos de evaluar y las evidencias de desempeño que a través de los productos esperados permitirán la evaluación (Verdejo, 2008). De acuerdo a esta misma autora, la evaluación por competencias requiere competencias evaluativas nuevas en los docentes, quienes deben generar instrumentos que "...deben cubrir los requisitos psicométricos de confiabilidad (reproducibilidad, las diferencias en los puntajes se deberán a diferencias en el desempeño), validez de contenido (que se mida lo que se pretende medir) y validez predictiva (predicción de la calidad y perfil de la práctica profesional)", junto con instrumentos que permitan la evaluación cualitativa para retroalimentar y fortalecer las competencias del Ser y del Saber Convivir.

En base a que la competencia es un desempeño en acción, que se aprendió a través de la mediación del docente con alta implicación del que aprende, la evaluación de competencias promueve la participación del sujeto evaluado, a través del ejercicio de la coevaluación y autoevaluación. Esto otorga al estudiante la capacidad de decidir sobre la calidad de su aprendizaje y decididamente favorece el ejercicio de la capacidad crítica y ética. Otro componente de la evaluación de competencias es la evaluación formativa o retroalimentación, la cual fue propuesta por Scriven en 1960, quien la define como aquella evaluación que es una permanente actividad de supervisión y acompañamiento. Es dinámica, flexible, continua y está al servicio de los objetivos pactados inicialmente y permite mejorar y corregir anticipadamente los errores y carencias.

De este modo la práctica evaluativa en el modelo por competencias requiere alejarse de la tradicional evaluación de pruebas escritas, hacia una evaluación comprensiva, holística que transparente la certificación del logro. 


\section{METODOLOGIA}

Los resultados que se presentan y discuten corresponden a la $1^{\mathrm{a}}$ etapa del proyecto S-2007-07 "Hacia un modelo curricular por competencias. Coherencia entre el discurso y las prácticas pedagógicas en el desarrollo de competencias genéricas. Un estudio de caso instrumental en carreras acreditadas", inscrito en la Dirección de Investigación y Desarrollo de la Universidad Austral de Chile (UACh). La investigación es de corte cualitativo, analítico-relacional, y su diseño corresponde a un estudio de caso instrumental (Stake, 1994) a desarrollar en tres etapas. El caso lo constituyen las carreras acreditadas de la UACh. El criterio de inclusión de las carreras fue que se encontrasen acreditadas al mes de noviembre del 2006 (fecha en que se envió el proyecto a Concurso). El universo quedó constituido por 10 carreras, se accedió a los programas de seis de ellas, por lo que el grupo quedó conformado por las carreras de: Enfermería - Medicina - Ingeniería Civil en Informática - Contador Auditor - Ingeniería Forestal y Biología Marina (ver tabla 1).

\section{Tabla 1}

Distribución numérica y porcentual de los programas revisados por Escuela

\begin{tabular}{|l|l|l|l|l|l|}
\hline Enfermería & 56 & $15,6 \%$ & Medicina & 62 & $17,3 \%$ \\
\hline Ing. Forestal & 62 & $17,3 \%$ & Ing. Civil en Informática & 83 & $23 \%$ \\
\hline Cont. Auditor & 56 & $15,6 \%$ & Biología Marina & 40 & $11,1 \%$ \\
\hline
\end{tabular}

Fuente: Consolidado de programas revisados DID S-2007-07.

El supuesto de investigación que guía este trabajo es que "Los procesos que sistematizan la instalación del currículo por competencias presentan grados de coherencia variada entre el discurso curricular formal y las prácticas pedagógicas debido a la manera tradicional de concebir la construcción curricular en la educación superior. Esta variación o desfase puede ser observada en: el diseño de los programas de asignatura en la presencia o no de relación entre las competencias genéricas a lograr en la asignatura, las metodologías que se planifican para su adquisición y las modalidades de la evaluación para certificar su logro. En el desarrollo de los programas ésta se puede observar a través de la contrastación de lo planificado y las prácticas pedagógicas observadas".

El objetivo general fue: Analizar la coherencia entre el discurso curricular formal -programas de asignaturas- y la práctica pedagógica de los diseños curriculares de carreras acreditadas de la UACh para el logro de competencias genéricas. Para verificar las competencias genéricas se usó el listado de competencias Tuning que adoptó la UACh (Decreto 271) y también las competencias sellos institucionales ${ }^{6}$. En tanto para establecer la forma en que se planteaba el logro de las competencias, las formas de desplegarlas

$6 \quad$ Ver Anexo 1. 
durante el curso y la manera de certificarles, se construyó y validó el "Indice de Coherencia Interna" (ICI) cuyo puntaje mínimo de corte fue de 30 puntos.

\section{EL INDICE DE COHERENCIA INTERNA, SU DISEÑO Y APLICACION (ver tabla 2)}

La búsqueda de la articulación de los componentes básicos Competencias genéricas/Objetivos, estrategias metodológicas y Estrategias evaluativas, contiene una primera intención: la búsqueda de coherencia que refleja en un plano técnico el quehacer de los constructores, pero también reflejan la concepción de Hombre, Mundo y Sociedad de quienes construyen. A partir de esta idea compartida por el equipo, se eligen las competencias genéricas justamente porque ellas incluyen el Aprender a ser y el Aprender a convivir juntos, las que el Informe Delors (1996) señala como parte de los pilares fundamentales de la educación. Es decir, el eventual desempeño con el cual los profesionales convivirán, mejorando, transformando o manteniendo el statu quo de la realidad en que se inserten.

En primer término se busca la explicitación de cada aspecto buscado, luego la forma en que éste se desarrolla en el programa y sus atributos. Por último se analiza la forma en que estos aspectos se relacionan unos con otros. Recibieron más alta ponderación aquellos aspectos que se relacionan directamente con las competencias/objetivos, metodologías y evaluación (resaltadas en cursiva) y fueron considerados trazadores para levantar el puntaje de corte. Se estableció una puntuación intermedia para aquellos aspectos que pudiesen estar medianamente explicitados en los programas. El puntaje máximo del ICI es 42 , determinándose como 30 el puntaje de corte que corresponde al logro del $71 \%$ del índice, lo que incluye el logro máximo de los ítems trazadores. Se realizó validación de contenido y de estructura por expertos, aplicándose previamente a 20 programas de otras asignaturas. Luego de ello se realizaron ajustes de formato, ponderación y redacción. Se establecieron tres rangos a partir del punto de corte Coherencia aceptable (30 a 33); Coherencia Excelente (34 a 37) y Coherencia Máxima (38 a 42). Lo mismo para los programas que no alcanzaron el mínimo: Incoherencia mínima (29 a 20); Incoherencia media (19 a 10) e Incoherencia máxima (10 y menos). Con el fin de disminuir el sesgo de diferentes interpretaciones, la revisión fue realizada por dos de las investigadoras. La aplicación del ICI se basó en el juicio experto de las revisoras especialistas en el modelo curricular por competencias. Fueron excluidos los programas de asignaturas que correspondiesen a asignaturas eminentemente prácticas.

\section{DISCUSION DE LOS RESULTADOS}

A partir del supuesto de que las carreras acreditadas presentan currículos orientados a competencias, los productos curriculares deberían contener los hitos estructurales básicos. Por esto se considera esta revisión como una primera y consistente aproximación para identificar el nivel de avance, apropiación y experticia alcanzado por los académicos en esta nueva forma de concebir la formación profesional. Dada la forma en que se instaló el modelo hubo desde el comienzo aprensiones y resistencias que de acuerdo a lo que sostienen Sumsion y Goodfellow (2004) se entretejieron en un sustrato de diferencias e imprecisiones conceptuales, carencia de metodologías para seleccionar las competencias, 
Tabla 2

Indice de Coherencia Interna

\begin{tabular}{|c|c|c|c|c|c|c|c|c|}
\hline \multicolumn{6}{|c|}{ El Programa tiene Descriptor o propósito } & 3 & & 0 \\
\hline \multicolumn{6}{|c|}{$\begin{array}{l}\text { En el Descriptor u otra parte del Programa revisado se señalan las } \\
\text { competencias genéricas a lograr por el estudiante }\end{array}$} & 4 & $\begin{array}{c}3 \\
\text { (Objetivos) }\end{array}$ & $\begin{array}{c}0 \\
\text { (No señala) }\end{array}$ \\
\hline \multicolumn{6}{|c|}{$\begin{array}{l}\text { En el Descriptor u otra parte del Programa revisado se señalan las } \\
\text { competencias específicas a lograr por el estudiante }\end{array}$} & 3 & $\begin{array}{c}1 \\
\text { Objetivo }\end{array}$ & $\begin{array}{c}0 \\
\text { No señala }\end{array}$ \\
\hline \multicolumn{6}{|c|}{$\begin{array}{l}\text { Las competencias a lograr corresponden a todos los ámbitos } \\
\text { Actitudinales - cognitivas - procedimentales }\end{array}$} & $\begin{array}{c}5 \\
(\mathrm{TRES})\end{array}$ & $\begin{array}{c}3 \\
(\mathrm{DOS})\end{array}$ & $\begin{array}{c}0 \\
{[\mathrm{UNA})}\end{array}$ \\
\hline \multicolumn{6}{|c|}{$\begin{array}{l}\text { La planeación y presentación general explicita contenidos } \\
\text { contextualizados }\end{array}$} & 4 & & 0 \\
\hline \multicolumn{6}{|c|}{$\begin{array}{l}\text { La planeación y presentación general explicita metodologías como: } \\
\text { ABP, Estudio de caso y Pasos prácticos o Proyectos o trabajo de } \\
\text { grupo o Investigación o debates, uso de TIC, portafolio }\end{array}$} & & & \\
\hline \multicolumn{2}{|c|}{$\begin{array}{l}\text { Predominio de ABP o Estudio } \\
\text { de caso o Pasos prácticos más } \\
\text { Proyectos o trabajo de grupo o } \\
\text { Investigación o debates o uso } \\
\text { de TIC o portafolio+ }\end{array}$} & \multicolumn{2}{|c|}{$\begin{array}{l}\text { ABP y/o Estudio } \\
\text { de caso y/o Pasos } \\
\text { prácticos más } \\
\text { Clase expositiva } \\
\text { más Proyectos o } \\
\text { trabajo de grupo } \\
\text { o Investigación } \\
\text { o debates, TIC, } \\
\text { portafolio }\end{array}$} & \multicolumn{2}{|c|}{$\begin{array}{l}\text { Predominio de } \\
\text { Clase expositiva } \\
\text { más Proyectos o } \\
\text { trabajo de grupo } \\
\text { o Investigación } \\
\text { o debates o } \\
\text { uso de TIC o } \\
\text { portafolio }\end{array}$} & 4 & 3 & $\begin{array}{c}0 \\
9 \\
\text { (no describe) }\end{array}$ \\
\hline \multicolumn{6}{|c|}{$\begin{array}{l}\text { Las metodologías planteadas se corresponde con el tipo de } \\
\text { competencia a lograr }\end{array}$} & $\begin{array}{c}5 \\
\text { (alto) }\end{array}$ & $\begin{array}{c}3 \\
\text { (media) }\end{array}$ & $\begin{array}{l}0 \\
(\mathrm{Ning}) \\
9\end{array}$ \\
\hline \multicolumn{6}{|c|}{$\begin{array}{l}\text { La estrategia de evaluación planteada corresponde al tipo de } \\
\text { competencias a lograr }\end{array}$} & $\begin{array}{c}5 \\
\text { (alto) }\end{array}$ & $\begin{array}{c}3 \\
(\text { media })\end{array}$ & $\begin{array}{c}0 \\
(\mathrm{Ning}) \\
9\end{array}$ \\
\hline \multicolumn{6}{|c|}{$\begin{array}{l}\text { La planeación general y presentación general explicita espacios para } \\
\text { la evaluación formativa }\end{array}$} & 2 & & 0 \\
\hline \multicolumn{6}{|c|}{$\begin{array}{l}\text { La planeación general y presentación general explicita estrategias de } \\
\text { evaluación de desempeños (ECOE, ABP, Debates, Informes) }\end{array}$} & \multirow[b]{2}{*}{5} & \multirow[b]{2}{*}{3} & \multirow[b]{2}{*}{$\begin{array}{l}0 \\
9\end{array}$} \\
\hline $\begin{array}{l}\text { Predominio de ECOE } \\
\text { o ABP o Debates o } \\
\text { Informes }\end{array}$ & $\begin{array}{l}\text { ECOE c } \\
\text { Debates } \\
\text { y prueb } \\
\text { escritas }\end{array}$ & $\begin{array}{l}\text { ABP o } \\
\text { o Informes } \\
\text { as orales o }\end{array}$ & $\begin{array}{l}\text { Pred } \\
\text { Prue } \\
\text { escr }\end{array}$ & $\begin{array}{l}\text { omin } \\
\text { bas } c \\
\text { tas }\end{array}$ & & & & \\
\hline \multirow{2}{*}{\multicolumn{2}{|c|}{ INDICE [ICI] }} & & AMC & $(+)$ & \multicolumn{4}{|c|}{$\begin{array}{l}\text { Coherencia Máxima } 38 \text { a } 42 \\
\text { Coherencia Excelente } 34 \text { a } 37 \\
\text { Coherencia Aceptable } 30 \text { a } 33\end{array}$} \\
\hline & & & \multicolumn{2}{|c|}{ TRAMO (-) } & \multicolumn{4}{|c|}{$\begin{array}{l}\text { Incoherencia Mínima } 29 \text { a } 20 \\
\text { Incoherencia Media } 19 \text { a } 10 \\
\text { Incoherencia Máxima } 9 \text { y menos }\end{array}$} \\
\hline
\end{tabular}


adopción de otros modelos sin problematizar las propuestas ni verificar el contexto y recursos necesarios para desarrollar las habilidades seleccionadas y, sobre todo, falta de evidencias que asegurasen que efectivamente éstas eran transferibles a otros contextos.

En esta publicación discutiremos algunos matices de los hallazgos producto de la revisión de programas de asignaturas, en espera de acceder con mayor profundidad al fenómeno en estudio. Con esta debida consideración, presentamos los resultados descriptivos y de distribución numérica y porcentual, provenientes de la revisión de los programas, junto con los relacionados a la aplicación del ICI y la descripción de las competencias referidas.

RESULTADOS DE LA APLICACION DEL ICI

Tabla 3

Distribución numérica y porcentual de programas con ICI $\geq$ a 30

\begin{tabular}{|c|c|c|}
\hline Total programas revisados & $\begin{array}{c}\text { Total Programas } \\
\text { ICI } \geq \text { a } 30\end{array}$ & $\begin{array}{c}\text { Porcentaje Total Programas } \\
\text { ICI } \geq \text { a } 30\end{array}$ \\
\hline 359 & 74 & $20 \%$ \\
\hline
\end{tabular}

Fuente: Consolidado de programas revisados DID S-2007-07.

De los 359 programas revisados, el 20\% (74 programas) alcanza puntajes similares o superiores a 30 puntos tras la aplicación del ICI (ver tabla 2). Esto da cuenta que al menos en la etapa del diseño existen tensiones que dificultan la articulación coherente entre al menos tres de los aspectos centrales del diseño de un programa: las competencias u objetivos a lograr, las metodologías diseñadas para ello y la estrategia evaluativa que determinará la calidad de tales logros. Esto es destacable en tanto que existe un modelo educativo declarado con instrumentos orientadores.

\section{Tabla 4}

Distribución numérica y porcentual de ICI $\geq$ a 30 por escuela

\begin{tabular}{|l|c|c|l|r|l|}
\hline Enfermería & 22 & $39 \%$ & Medicina & 16 & $17,3 \%$ \\
\hline Ing. Forestal & 10 & $16 \%$ & Ing. Civil en Informática & 8 & $9,6 \%$ \\
\hline Contador Auditor & 6 & $10,7 \%$ & Biología Marina & 12 & $30 \%$ \\
\hline
\end{tabular}

Fuente: Consolidado de programas revisados DID S-2007-07.

En cuanto a los hallazgos por Escuela, destaca la Escuela de Enfermería que dentro del total de programas revisados alcanza el mayor porcentaje de programas con ICI $\geq$ a 30, 39\% (ver tabla 3), a lo que le sigue la Escuela de Biología Marina con un 30\% 
y Medicina con un 17,3\%. Estos resultados se pueden explicar porque estas escuelas han revisado y modificado sus mallas curriculares, producto de proyectos MECESUP o cambios curriculares motivados por la institución. Es necesario señalar que las otras carreras seleccionadas para este estudio se encuentran en distintos grados de avance de revisión de sus planes de estudio, lo que seguramente se verá reflejado en los resultados de las siguientes fases de este proyecto. El caso de Enfermería se discute en el artículo "Factores que influyen en la declaración de competencias genéricas en los programas de estudio: el caso de la Escuela de Enfermería de la Universidad Austral de Chile. Resultados preliminares" que también prepara nuestro grupo de trabajo.

La distribución porcentual de programas que alcanzan coherencia deja en evidencia el bajo porcentaje de programas cuyo diseño explicita -de acuerdo a los criterios planteados- la articulación lógica entre las competencias, las metodologías para desarrollar esas capacidades y las estrategias de evaluación que permitan dar cuenta del logro de ellas al finalizar el periodo estipulado para su logro. El aspecto en el que se presenta la menor coherencia es en la relación objetivos/competencias declaradas versus estrategias de evaluación y en un grado menor, pero no menos importante en la relación objetivos/competencias y metodologías.

En las estrategias evaluativas se aprecia el predominio de las pruebas escritas y exámenes orales, como mecanismos de certificación de los logros, lo que se relaciona con la certificación de conocimientos que implican habilidades cognitivas (Bloom, 1956) que de acuerdo a lo revisado por Anderson y Krathwohl (2001) favorece la memorización y no necesariamente desempeños en la acción. En tanto la modalidad de la evaluación formativa aparece explicitada en menos del $10 \%$ de los programas y se destaca la casi nula participación de los estudiantes en la evaluación.

En las estrategias metodológicas priman sobre el $70 \%$ de los programas las sesiones expositivas, seguidas del trabajo de grupo, como metodologías más utilizadas. Esto contrasta con las metodologías que se declaran en los programas con ICI (+), en ellos se aprecia alto uso del ABP; Trabajos de Proyectos; Portafolio y Actividades fuera del aula como visitas a centros laborales. Un análisis más detallado de la relación específica entre las variables del estudio, será desarrollado en un próximo artículo.

\section{COMPETENCIAS GENERICAS}

Se desglosa en las más explicitadas y menos explicitadas.

Tabla 5

Distribución numérica y porcentual de las competencias genéricas más explicitadas

\begin{tabular}{|l|c|c|}
\hline Competencia Tuning & Total & $\%$ \\
\hline Capacidad de aplicar los conocimientos en la práctica & 166 & 46 \\
\hline Conocimiento sobre el área de estudio y la profesión & 158 & 44 \\
\hline Capacidad de abstracción, análisis y síntesis & 155 & 43 \\
\hline Habilidades en el uso de las tecnologías de la información y la comunicación & 75 & 20 \\
\hline Habilidades para buscar, procesar y analizar información procedente de fuentes diversas & 71 & 20 \\
\hline Capacidad para tomar decisiones & 70 & 19 \\
\hline
\end{tabular}

Fuente: Check list de Competencias Genéricas en programas de asignaturas. 
Es destacable, en lo que respecta a este listado, que dentro de las competencias genéricas más enunciadas aparezca en primer lugar la Capacidad para aplicar los conocimientos en la práctica. Esto da cuenta de la intencionalidad a partir del discurso formal de desarrollar efectivamente esas capacidades, relación de los conocimientos con el contexto profesional. A ello le sigue el Conocimiento sobre el área de estudio y la profesión, lo que da cuenta explícitamente del valor que sigue ocupando el conocimiento como tal, aunque en este caso está contextualizado a las áreas de la profesión. De acuerdo a Bloom y Anderson \& Krathwohl, las habilidades reforzadas en esta competencia tienen que ver con la memorización, recuerdo y reproducción de esos conocimientos. Por último, la tercera competencia más enunciada en los programas es la Capacidad de abstracción, análisis y síntesis, que siendo habilidades intelectuales permiten analizar, enjuiciar y crear nuevas respuestas a los problemas.

Si se analizan en conjunto, los programas revisados al menos en el plano discursivo del programa escrito privilegian competencias que permiten el uso pertinente de los conocimientos en contextos diferentes y además proveen de las herramientas para mejorar la realidad solucionando problemas. Sin embargo esto también es lo que tradicionalmente ha sido desarrollado en el proceso de enseñanza y aprendizaje de los programas basados en objetivos. Claramente las formas en que se llevan a cabo, el sentido que las competencias tienen en relación al perfil de egreso, la forma en que son evaluadas y la centralidad que adquiere el que aprende hacen la distinción.

\section{Tabla 6}

Distribución numérica y porcentual de las competencias genéricas menos explicitadas

\begin{tabular}{|l|c|c|}
\hline Competencia Tuning & Total & $\%$ \\
\hline $\begin{array}{l}\text { Habilidades interpersonales } \\
\text { Habilidades para trabajar en contextos internacionales }\end{array}$ & 3 & 0,83 \\
\hline $\begin{array}{l}\text { Compromiso con su medio sociocultural. Valoración y respeto por la } \\
\text { diversidad y multiculturalidad } \\
\text { Compromiso ético }\end{array}$ & 4 & 0,84 \\
\hline Capacidad de aprender y de actualizarse permanentemente & 5 & 1,4 \\
\hline Compromiso con la preservación del medio ambiente & 9 & 2,5 \\
\hline Capacidad crítica y autocrítica & 11 & 3,1 \\
\hline
\end{tabular}

Fuente: Check list de Competencias Genéricas en programas de asignaturas.

En primer lugar aparecen dos competencias menos explicitadas: las habilidades interpersonales y la habilidad para trabajar en contextos internacionales. De acuerdo a lo que señala Caballo (1986), la conducta socialmente habilidosa es un conjunto de conductas realizadas por un individuo en un contexto interpersonal que expresa sentimientos, actitudes, deseos, opiniones o derechos de un modo adecuado a la situación, respetando esas conductas en los demás, y que generalmente resuelve los problemas inmediatos de 
la situación mientras reduce la probabilidad de que aparezcan futuros problemas. Estas habilidades son vitales a la hora de trabajar en equipo o desarrollar procesos creativos. Suponemos que su no explicitación podría obedecer a que aún no adquiere el rango de "enseñable sistemáticamente", dado que o se le considera una capacidad innata o se supone que a través de la acción del docente como modelador se "aprenderá". Datos aún no procesados de las entrevistas de los docentes participantes del estudio, así nos lo hacen suponer. Y en tanto a la habilidad para el trabajo en contextos internacionales, probablemente obedezca a que es una práctica incipiente al menos en nuestro medio.

Las dos siguientes competencias relacionadas con el compromiso con el medio y el quehacer ético nos parecen ausencias graves, dado que éstas desarrollan y maduran un profesional íntegro y relacionado con el medio y sobre todo con las personas o comunidades donde ejercerá. En un país que mantiene inequidades en la distribución de los ingresos y en el que existen grupos discriminados que no tienen acceso a los beneficios del sistema social, no se entiende un profesional que se forme sin estos énfasis. Ahora bien, si se revisan los perfiles de egreso de las carreras estudiadas, todas ellas señalan el compromiso ético y el respeto por los demás, todo lo cual nos permite visualizar incoherencia en este plano. Ahora bien, algunas carreras han generado cursos o módulos independientes de Ética y de desarrollo de habilidades, lo que es una forma desde el currículo de apuntar al logro de estas capacidades y compromisos, sin embargo la característica de transversalidad que ambas tienen hacen necesaria que se liguen de forma explícita con los conocimientos y contextos disciplinares, es decir, en todas las asignaturas. No se aprende a ser ético en un curso de Ética. En un curso de Ética, cognitivamente se comprenden los principios, postulados, conceptos, pero, se ES ético en contexto ante las tensiones reales del mundo laboral y se ES hábil en las relaciones interpersonales, interactuando efectivamente en situaciones concretas.

La Competencia que se refiere a aprender y actualizarse permanente, nos parece una omisión en el texto. Es necesario decir que cada competencia se buscó tal cual su enunciación o muy cercana a ella, pero se evitó la inferencia a partir de otros datos. Por este motivo, suponemos que este dato no representa, al menos, al grupo de asignaturas que alcanzan el punto de corte del ICI, dado que en ellas las metodologías por sí mismas fomentan el autoaprendizaje.

Para la competencia relacionada con la preservación del medio ambiente, ésta sólo parece en los cursos relacionados con las carreras de Biología Marina e Ingeniería Forestal. Las otras carreras no explicitan esta capacidad, dando cuenta de que aún no se considera la noción de integralidad en la formación. Desde cualquier ámbito profesional se puede cuidar el medio ambiente, más hoy en día en que a causa justamente de manejos profesionales que no consideraron la preservación o el desarrollo sustentable, se han deteriorado e incluso perdido atributos del medio natural que repercuten en el desarrollo de las comunidades y que significan a la formación profesional tener miradas más amplias y prospectivas, para no desaparecer por la ausencia de campus de ejercicio o mejorar su ubicación en el "situs" profesional.

En cuanto a la ausencia en los programas de la explicitación del desarrollo de la capacidad crítica o autocrítica, nos parece una ausencia inquietante. Ciertamente lo "crítico" ha estado siempre relacionado a lo conflictivo y lo conflictivo suele evitarse. Fortalecer el pensamiento crítico en los estudiantes también implica hacerse cargo de esa capacidad, lo que puede llevar al riesgo de que el docente sea el blanco de ese razonamiento crítico. 
$\mathrm{Su}$ ausencia puede obedecer a que se le asuma "no enseñable" o que se le considere adquirida en forma tácita a través de la observación.

Un profesional que ha sido formado en la lógica del Pensamiento crítico y la autocrítica debería acercarse a lo que señala el Informe Delphi (Facione 2006), citado por Alfaro, (2009:8):

"El pensador crítico ideal es habitualmente inquisitivo, autoinformado, confía en los argumentos, es de mente abierta, flexible, imparcial en la evaluación, honrado al afrontar los sesgos personales, prudente al formular juicios, dispuesto a reconsiderar, claro sobre los temas, ordenado en los asuntos complejos, diligente en la búsqueda de información relevante, razonable en la selección de criterios y persistente en la búsqueda de resultados que son tan precisos como el sujeto y las circunstancias de la indagación lo permiten".

Por esta razón consideramos que esta es una importante tarea pendiente de las acciones que el docente debe implementar y mediar para su aprendizaje, y que tal como señala Barnett (2002) la supercomplejidad implica una pedagogía que no se quede en la mera transmisión de conocimientos o adquisición de destrezas, sino que el estudiante tenga un espacio pedagógico propio, en el cual pueda exponer y desarrollar su propia voz.

En lo que respecta a las competencias sellos institucionales sólo se describen en el $2 \%$ de los programas revisados, siendo la más frecuente la que se relaciona con Responsabilidad Social. Asumimos que esto también obedece a la no explicitación o al hecho de que se consideren inmersas dentro de otras, es decir, tácitas.

Un aspecto emergente y que dificultó la revisión es que los programas revisados presentaban diferentes formatos y grados de profundidad. Se cotejaron al menos 20 formatos distintos no sólo entre carreras sino que al interior de ellas mismas. El 70\% de los programas revisados contenían estructuras similares, conservando en su desarrollo los aspectos recomendados en su diseño. En tanto, el 30\% restante aportaba menos información que la necesaria para su análisis, siendo los aspectos relacionados con las metodologías y la evaluación los más lesionados. Ambos aspectos son discutidos en los artículos de nuestro grupo "Valor del programa como hipótesis del itinerario formativo en carreras que promueven competencias. Resultados preliminares" de Sandoval y cols.; y "Sistemas de gestión curricular en la UACh" de Pavié y cols.

\section{CONCLUSIONES}

La búsqueda de la coherencia interna de los programas -a través de la búsqueda de las competencias genéricas- comprobó la primera parte de nuestro supuesto de investigación. Se aprecian con claridad contradicciones en los programas de asignaturas. De acuerdo a ello, concluimos que es claro el proceso de transición. Tomando a Khun, aún no se resuelve qué prácticas anteriores conservar y cuáles son las nuevas para incorporarlas. Pendiente entonces queda la apropiación curricular como tal que permita asumir que se ha instalado una nueva modalidad de construir currículo. El hallazgo de un alto porcentaje de programas que no alcanzan el puntaje de corte con el ICI es un dato a considerar con resguardo -como antes señalamos-, dado la multiplicidad de otros factores que puedan estar interviniendo en los diseños, desde los antecedentes que se consideran relevantes para ser incorporados, al nivel de dominio de quien los diseña, el valor que le atribuye al programa como herramienta que mejora los aprendizajes, el uso 
de otros documentos al interior de la asignatura que no se mencionan en el programa e incluso el tiempo de que se dispone para llevar a cabo tal diseño, junto con el hecho de que existe un activo proceso de reorganización curricular en nuestra casa de estudios y capacitaciones diseñadas para ello desde la estructura central, junto con la introducción de un formato estándar para el diseño de programas.

En torno a la capacitación docente en los temas de diseño curricular, metodologías y evaluación para poder impactar en los resultados de aprendizaje se dispone de abundante sustento teórico en la literatura (Perrenoud P., 2004; Zabalza, M., 2004-2007; Villar, L., 2004; Zabala, A., 2007). Pero de acuerdo a Villar (2004), la capacitación debe acompañarse de un proceso activo de seguimiento cuyo acento es la enseñanza reflexiva para dar cuenta de un docente con un rol practicador reflexivo. De acuerdo a lo que sostiene Schön (1992), la práctica reflexiva, permite al docente mirar su quehacer antes, durante y después, rescatando el alto valor de la experiencia práctica. De este modo la confección del programa puede constituirse en un hecho valorado por su impacto en el estudiante y no sólo ser considerado un trámite más a llevar a cabo.

La capacitación reflexiva y acompañada podría favorecer por otra parte el necesario proceso de cambio conceptual requerido para abandonar las concepciones anteriores y considerar factibles los nuevos conocimientos en consideración a los postulados de Pozo (1989), quien sostiene que la adscripción a nuevos modelos representacionales requiere por un lado el uso de lenguajes o códigos conocidos y la construcción activa de la nueva estructura de conocimiento. "No se trata de enseñar a los alumnos esos esquemas y principios generales, sino ayudarles a construir esos esquemas en dominios o contextos específicos, como posibles respuestas a determinados problemas, para que luego se generalice o transfiera a otros nuevos" (1999, pp. 516). Por otro lado, Martin, E. \& Lueckenhausen, G. (2005) reportan en un estudio llevado a cabo en una universidad australiana, que el seguimiento durante un semestre a 31 académicos de distintas disciplinas, que los cambios en la forma de enseñar son acompañados de cambios en la esfera emocional, y sostienen que este aspecto ha sido poco considerado en la universidad y lo relevan como un aspecto importante a considerar para impactar en los resultados de aprendizajes.

\section{BIBLIOGRAFIA}

MECESUP (s.f.). Acerca del programa MECESUP. Recuperado el 31 de mayo de 2006, del sitio web del Ministerio de Educación: www.mineduc.cl.

Centro Nacional de Arbitrajes CNA (2009). Cláusulas arbitrales. Recuperado el 20 de octubre de 2009, del Sitio http://www.cna.cl/index-1.php?id=clausulas.

Vigotzky, L.S. (2001). Psicología pedagógica: un curso breve. Buenos Aires: Aique, 2001. 502 p.

Ausubel, D. (1976). Psicología educativa: un punto de vista cognoscitivo. México Trillas c1976. 769 p.

Hoogveld A., Paas F., \& Jochems, W. (2003). Application of an instructional systems design approach by teachers in higher education: individual versus team design. Teaching and Teacher Education, Volume 19, Issue 6, August 2003, pp. 581-590.

Márquez, M. (2008). Tensiones y demandas a los constructores curriculares de la educación universitaria. El escenario de los proyectos de Mejoramiento de la Calidad de la Educación Superior (MECESUP), en Chile. Revista de estudios y experiencias en educación REXE. Volumen $7, \mathrm{~N}^{\circ} 13$. 
Coll, C. (1987). Psicología y currículum: una aproximación psicopedagógica a la elaboración del currículum escolar. Buenos Aires: Argentina, Paidós.

Zabalza, M.A. (2000). Diseño y desarrollo curricular (5ta. Ed.). Madrid: Narcea. 310 p.

Barnett, R. (2002). Claves para entender la universidad en una era de supercomplejidad. España: Barcelona, Pomares.

Brunner, J. J., \& Uribe, D. (2007). Mercados universitarios: el nuevo escenario de la educación superior. Santiago: U. Diego Portales.

Zabalza, M. A. (2000). Los planes de estudio en la universidad. Algunas reflexiones para el cambio. Revista Fuentes. Universidad de Santiago de Compostela.

Aristimuño, A. (2008). Las competencias en la educación superior: ¿demonio u oportunidad?

Departamento de Educación, Universidad Católica del Uruguay. Recuperado el 14 de octubre de 2009, del Sitio http://www.cna.cl/index-1.php?id=clausulas.

Sandoval, J. \& Márquez, M. (2007). Análisis de Prácticas Docentes y Pedagógicas en las Escuelas de Tecnología Médica, Enfermería, Obstetricia y Puericultura Asesorías para el Proyecto MECESUP AUS 0209. (Contraparte técnica).

Universidad Austral de Chile (2009). Objetivos Estratégicos Plan Estratégico 2008-2011. (16-17).

Jabif, L. (2007). Orientaciones prácticas para docentes, la docencia universitaria bajo un enfoque de competencias. Universidad Austral de Chile.

Universidad Austral de Chile (2007). Modelo educacional y enfoque curricular de la UACh.

Le Boterf, G. (2000). Ingeniería de las competencias. Barcelona: Ed. Gestión.

Universidad Austral de Chile (2005). Decreto 271 Orientaciones Curriculares de Pregrado.

McClelland, D. (1973). Medir la competencia en lugar de la inteligencia. En: Nuevo ataque contra la igualdad de oportunidades Cociente intelectual y estratificación social de Gartner, A., Greer, C. \& Riesssman, F. (1999). Sitio http://books.google.cl/books?id=v8X4gK5oAewC\&pg=PA1 $24 \& l p g=P A 124 \& d q=$ mcclelland+test+de+inteligencia $+1973 \&$ source $=$ bl\&ots $=$ WEGFcIKxbF\& sig=4SkqzdXE6W-TPvI3gNsjOjP2Vp0\&hl=es\&ei=Qw8xS5jjBZG1tgeirJ2RCQ\&sa=X\&oi=bo ok_result\&ct=result\&resnum $=2 \& v e d=0 C A w Q 6 A E w A Q \# v=$ onepage $\& q=$ mcclelland $\% 20$ test $\% 20$ de\%20inteligencia\%201973\&f=false.

Schmal, R. y Ruiz Tagle, A. (2007). Una metodología para el desarrollo de competencias. Ingeniare, Revista chilena de ingeniería, vol. $16 \mathrm{~N}^{\circ} 1,2008$, pp. 147-158.

Cowan, J. (1998). On becoming an innovative university teacher. Buckingham: Open University press. En Knigtht, P. (2006). El profesorado de Educación superior, formación para la excelencia, Knight, P. (2006). Madrid: Narcea.

Zabala, A. \& Arnau, L. (2007). Cómo aprender y enseñar competencias, once ideas claves. Barcelona: Graó.

Smith, D., Hedley, P., \& Molloy, M. (2008). Design learning: a reflective model Design Studies Volume 30, Issue 1, January 2009, pp. 13-37.

Timmerman, B., Strickland, D. \& Carstensen, S. (2008) Curricular reform and inquiry teaching in biology: where are our efforts most fruitfully invested? Integrative and Comparative Biology 200848 (2): 226-240, doi:10.1093/icb/icn064.

Herrera, M. (2009). "La autoevaluación en las prácticas pedagógicas de los alumnos del profesorado en enseñanza primaria”. Facultad de Ciencias de la Educación Universidad Nacional de Comahue, Argentina. Recuperado el 24 de septiembre de 2009, del Sitio http://www.rieoei. org/deloslectores/Herrera.pdf.

Scriven, M. (1960). Metodología de Evaluación formativa. En Evaluación sistemática: guía teórica y práctica. (1987). Stuffllebeam, D. \& Shinkfield, A. Bardelona: Paidós.

Verdejo, P. (2008). Modelo para la Educación y Evaluación por Competencias (MECO) ACET, Aseguramiento de la Calidad en la Educación y en el Trabajo, México. Recuperado el 4 de noviembre de 2009, del Sitio http://www.6x4uealc.org/site2008/p01/11.pdf.

Informe Delor (1996). "La Educación Encierra Un Tesoro". Capítulo IV, Ediciones UNESCO. 
Sumsion, J. \& Goodfellow, J. (2004). Identifying generic skills through curriculum mapping: a critical evaluation. Higher Education Research \& Development. Vol. 23, № 3 329-346.

Bloom, B. et al. (1971). Taxonomía de los objetivos de la educación: la clasificación de las metas educacionales: manuales I y II. Traducción de Marcelo Pérez Rivas Buenos Aires: Centro Regional de Ayuda Técnica: Agencia para el Desarrollo Internacional (A.I.D).

Anderson, L.W., \& Krathwohl, D. (2001). A Taxonomy for Learning, Teaching, and Assessing: A Revision of Bloom's Taxonomy of Educational Objectives. New York: Longman, 2001.

Caballo, V. (1986). Relaciones Interpersonales. Definición. Recuperado el 12 de diciembre de 2009, del Sitio http://www.down21.org/act_social/relaciones/1_h_sociales/definic_clasific.htm.

Facione, P. (1990). The Delphi Report Summary. Critical Thinking: A Statement of Expert Consensus for Purposes of Educational Assessment and Instruction Recuperado el 19 de diciembre de 2009, del Sitio http://assessment.aas.duke.edu/documents/Delphi_Report.pdf.

Alfaro, R. (2009). Pensamiento crítico y juicio clínico en Enfermería. (p. 8) España: Elsevier.

Perrenoud, Ph. (2004). Desarrollar la práctica reflexiva en el oficio de enseñar. Barcelona: Graó.

Schön, D. (1992). La formación de profesionales reflexivos: hacia un nuevo diseño de la enseñanza y el aprendizaje en las profesiones Barcelona: Paidós.

Villar, L. (2004) Programa para la mejora de la docencia universitaria. Madrid: Pearson Prentice Hall.

Pozo, J. I., (1989). Teorías cognitivas del aprendizaje. Madrid: Morata.

Martin, E. \& Lueckenhausen, G. (2005). How University Teaching Changes Teachers: Affective as Well as Cognitive Challenges Higher Education, Vol. 49, No. 3, Issues in Teaching and Learning from a Student Learning Perspective: A Tribute to Noel Entwistle (Apr., 2005), pp. 389-412. 
ANEXO 1

LISTADO DE COMPETENCIAS GENÉRICAS TUNING - SELLO UACH.

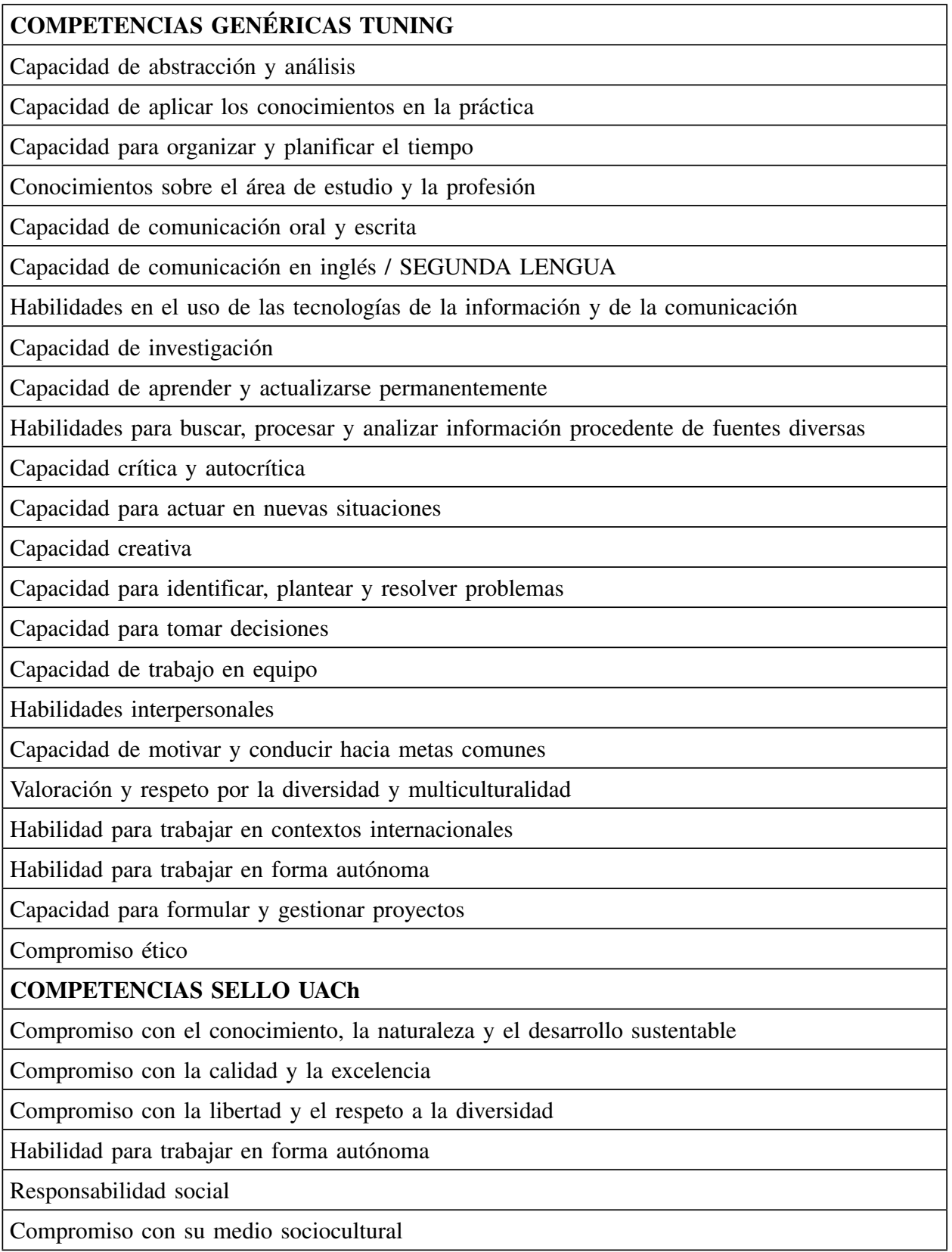

Fuente: Decreto 271, UACh, 2005. 